\title{
Pipelines Designing By Using Hydraulic Criteria Equations
}

\author{
Gencho Popov ${ }^{l, *}$, Kliment Klimenntov ${ }^{l}$ and Boris Kostov ${ }^{l}$ \\ ${ }^{1}$ University of Ruse, Department "Heat, Hydraulics and Environmental Engineering”, Ruse, \\ Bulgaria
}

\begin{abstract}
This work intend an original methodology, newly established by the authors, to design typical pipe systems by using hydraulic criteria equations, excluding the determination of the relevant Darcy factor. Therefore, it can be eliminated the necessity for searching iterative solutions of such problems. The established equations are easy to use, in terms of mathematical processing, which guarantees their proper application in solving engineering problems. It is considered for the proposed physical relations (equations) concerning the determination of the pipe system resistance characteristics to be highly useful in engineering practice, as for example: in determining a hydraulic machine work regime, used in a system transporting fluids, as well as in performing a synthesis and analysis of such systems or in accomplishing an energy analysis of pipe systems. A comparative analysis between the results found after applying the classical methods of calculations and by using a licensed specialized software product indicates that a highly sufficient (for engineering calculations) overlapping is achieved.
\end{abstract}

\section{Introduction}

Performing hydraulic calculations of a pipe system is one of the main tasks in designing a system, used to transport fluids. It is well-known that a major difficulty is to determine the resistance coefficients and more specifically the relevant Darcy friction factors $-\lambda_{i}$. In [1] and [2], the authors present a method for avoiding difficulties in determining this coefficient.

It is also known $[3,4]$ that the basic tasks concerning the hydraulic estimation (analysis) of a pipe system are to determine the required pressure (pressure drop between the pipe inlet and outlet) $\mathrm{p}$ for ensuring a given flow rate $Q$, or to determine the flow rate $Q$ in case that the pressure $\mathrm{p}$ is known.

The method for solving the above problems concerning simple pipes (no branches) with constant diameters d, without determining the friction factor, is

\footnotetext{
*Corresponding author: gspopov@uni-ruse.bg
} 
presented in $[1,2]$. By using the Dimensional Analysis (DA) it is established different criteria equations including three dimensionless complexes ( $\pi$ - criterions), each containing only one of the analyzed parameters - pressure $p$, flow rate $Q$ or diameter $d$. These equations, obtained in [2], respectively are:

$$
\begin{aligned}
& \pi_{p}=p \frac{\rho \Delta^{3}}{L \mu^{2}}, \\
& \pi_{Q}=Q \frac{\rho}{\mu \Delta}, \\
& \pi_{d}=\frac{\Delta}{d},
\end{aligned}
$$

where $p$ is the pressure difference between the pipe inlet (start) and outlet (end); $Q$ - the ensured flow rate; $L$ - the referred pipe length; $d$-the pipe diameter; $\Delta$ - the pipe walls roughness; $\rho, \mu$ - the fluid density and dynamic viscosity.

For obtaining the following hydraulic criteria equations a methodology, proposed in [2], is used:

$$
\begin{aligned}
& \pi_{p}=A \pi_{Q}^{a} \pi_{d}^{b} \\
& \pi_{Q}=B \frac{\pi_{p}^{e}}{\pi_{d}^{f}},
\end{aligned}
$$

where the values of the coefficients $A$ and $B$, as well as the exponents $(a, b, e, f)$, are found by using the pre-cited method.

Using the above equations enables determining the main hydraulic parameters for each "i" pipe. The flow rate $Q_{i}$ can be estimated by using the flow rate criterion $\pi_{Q}$ (4) for the current pipe:

$$
Q_{i}=\pi_{Q, i} \Delta_{i} \frac{\mu}{\rho} .
$$

Analogically, the pressure $p_{i}$ for each pipe is determined by using the pressure criterion (3):

$$
p_{i}=\pi_{p, i} \frac{L_{i}}{\Delta_{i}^{3}} \frac{\mu^{2}}{\rho} .
$$

A significant detail in accomplishing an analysis of a pipe system, used to transport fluids, is the obtaining of its resistance characteristic, which is often presented in the following way:

$$
p=k Q^{\alpha},
$$

where $\mathrm{k}$ is the coefficient of the pipe system resistance characteristics, $\mathrm{m}-$ is an exponent, which in case of completely rough pipes is equal to two $(\alpha=2)$.

The main purpose of this work is to establish an easily applicable in engineering 
calculations methodology for accomplishing hydraulic pipe sizing and analysis of typical pipe systems - pipes connected in parallel, in series or combined, by using the criteria equations (4) and (5).

\section{Main types of pipe systems transporting fluids}

This paragraph represents the application of equations (1) and (2) in performing hydraulic analysis of several types of typical complex pipe systems.

\subsection{Pipe systems containing pipes connected in parallel}

In case of pipes connected in parallel the following physical relations are valid:

- $\quad$ summing the flow rates ensured in each pipe should be equal to the system total flow rate $Q$ :

$$
Q_{1}+Q_{2}+\ldots+Q_{n}=Q
$$

- the pressure loss in each of the parallel connected pipes is equal the system total pressure loss:

$$
p_{1}=p_{2}=\ldots=p_{n}=p
$$

\subsubsection{Different type of pipes connected in parallel}

In general, a pipe system contains different types of pipes. In this regard, the absolute equivalent wall roughness $\Delta_{i}$ for each pipe has a different value.

By using equation (6) for the ensured flow rate in each pipe it is obtained that:

$$
\pi_{Q, 1} \Delta_{1} \frac{\mu}{\rho}+\pi_{Q, 2} \Delta_{2} \frac{\mu}{\rho}+\ldots+\pi_{Q, n} \Delta_{n} \frac{\mu}{\rho}=\pi_{Q} \Delta \frac{\mu}{\rho},
$$

where $\pi_{Q, i}$ and $\Delta_{i}(i=1 \ldots n)$ respectively are the flow rate criterion and wall roughness for each pipe, and $\pi_{Q}$ and $\Delta$ - represent the same parameters but concerning the common pipe (for example, it may be the pipe just before the splitting or after the collecting point of the analyzed parallel connected pipes).

Indicating that the fluid transported through each pipe has the same properties $\mu / \rho=$ const. , it may be concluded that:

$$
\pi_{Q, 1} \Delta_{1}+\pi_{Q, 2} \Delta_{2}+\ldots+\pi_{Q, n} \Delta_{n}=\pi_{Q} \Delta .
$$

Equation (7), enabling the estimation of the pressure loss in each of the parallel connected pipes by taking into the account the relevant criterion - $\pi_{p, i}$, is involved in (10). Indicating that $\mu^{2} / \rho=$ const. it may be stated:

$$
\pi_{p, 1} \frac{L_{1}}{\Delta_{1}^{3}}=\pi_{p, 2} \frac{L_{2}}{\Delta_{2}^{3}}=\ldots=\pi_{p, n} \frac{L_{n}}{\Delta_{n}^{3}} .
$$


The criteria equation (1), applied for each pipe, is used to determine the relevant criterions - $\pi_{p, i}$.

For presenting the parameters $\pi_{p, i}$ it is used the criteria equation (4). Indicating that the constant $A$, given in (4), is the same for each pipe, equation (11) may be presented as it follows:

$$
\pi_{Q, 1}^{a} \pi_{d, 1}^{b} \frac{L_{1}}{\Delta_{1}^{3}}=\pi_{Q, 2}^{a} \pi_{d, 2}^{b} \frac{L_{2}}{\Delta_{2}^{3}}=\ldots=\pi_{Q, n}^{a} \pi_{d, n}^{b} \frac{L_{n}}{\Delta_{n}^{3}} .
$$

Using the equations (12) and (14) enables the establishing of the following system of equations:

$$
\mid \begin{gathered}
\pi_{Q, 2}^{a} \pi_{d, 2}^{b} \frac{L_{2}}{\Delta_{2}^{3}}=\pi_{Q, 1}^{a} \pi_{d, 1}^{b} \frac{L_{1}}{\Delta_{1}^{3}} \\
\pi_{Q, 3}^{a} \pi_{d, 3}^{b} \frac{L_{3}}{\Delta_{3}^{3}}=\pi_{Q, 1}^{a} \pi_{d, 1}^{b} \frac{L_{1}}{\Delta_{1}^{3}} \\
\ldots \\
\pi_{Q, n}^{a} \pi_{d, n}^{b} \frac{L_{n}}{\Delta_{n}^{3}}=\pi_{Q, 1}^{a} \pi_{d, 1}^{b} \frac{L_{1}}{\Delta_{1}^{3}} \\
\pi_{Q, 1} \Delta_{1}+\pi_{Q, 2} \Delta_{2}+\ldots+\pi_{Q, n} \Delta_{n}=\pi_{Q} \Delta
\end{gathered} .
$$

Indicating that a pipe relative roughness criterion is $\pi_{d}=\frac{\Delta}{d}$, it can be concluded:

$$
\pi_{d, i}^{b} \frac{L_{i}}{\Delta_{i}^{3}}=\Delta_{i}^{b-3}\left(\frac{L_{i}}{d_{i}^{b}}\right),
$$

and respectively the system of equations (15) has to be transformed in the following way: 


$$
\mid \begin{gathered}
\pi_{Q, 2}^{a} \Delta_{2}^{b-3} \frac{L_{2}}{d_{2}^{b}}=\pi_{Q, 1}^{a} \Delta_{1}^{b-3} \frac{L_{1}}{d_{1}^{b}} \\
\pi_{Q, 3}^{a} \Delta_{3}^{b-3} \frac{L_{3}}{d_{3}^{b}}=\pi_{Q, 1}^{a} \Delta_{1}^{b-3} \frac{L_{1}}{d_{1}^{b}} \\
\cdots \\
\pi_{Q, n}^{a} \Delta_{n}^{b-3} \frac{L_{n}}{d_{n}^{b}}=\pi_{Q, 1}^{a} \Delta_{1}^{b-3} \frac{L_{1}}{d_{1}^{b}} \\
\pi_{Q, 1} \Delta_{1}+\pi_{Q, 2} \Delta_{2}+\ldots+\pi_{Q, n} \Delta_{n}=\pi_{Q} \Delta
\end{gathered}
$$

Then, this system of equations may be presented as it follows:

$$
\mid \begin{gathered}
\left(\pi_{Q, 2} \Delta_{2}\right)^{a} \Delta_{2}^{b-a-3} \frac{L_{2}}{d_{2}^{b}}=\left(\pi_{Q, 1} \Delta_{1}\right)^{a} \Delta_{1}^{b-a-3} \frac{L_{1}}{d_{1}^{b}} \\
\left(\pi_{Q, 3} \Delta_{3}\right)^{a} \Delta_{3}^{b-a-3} \frac{L_{3}}{d_{3}^{b}}=\left(\pi_{Q, 1} \Delta_{1}\right)^{a} \Delta_{1}^{b-a-3} \frac{L_{1}}{d_{1}^{b}} \\
\ldots \\
\left(\pi_{Q, n} \Delta_{n}\right)^{a} \Delta_{n}^{b-a-3} \frac{L_{n}}{d_{n}^{b}}=\left(\pi_{Q, 1} \Delta_{1}\right)^{a} \Delta_{1}^{b-a-3} \frac{L_{1}}{d_{1}^{b}} \\
\pi_{Q, 1} \Delta_{1}+\pi_{Q, 2} \Delta_{2}+\ldots+\pi_{Q, n} \Delta_{n}=\pi_{Q} \Delta
\end{gathered} .
$$

After being mathematically transformed the above equation may be presented in the following way:

$$
\mid \begin{gathered}
\pi_{Q, 2} \Delta_{2}=\left(\pi_{Q, 1} \Delta_{1}\right)\left(\frac{L_{1}}{L_{2}}\right)^{\frac{1}{a}}\left(\frac{d_{2}}{d_{1}}\right)^{\frac{b}{a}}\left(\frac{\Delta_{1}}{\Delta_{2}}\right)^{\frac{b-a-3}{a}} \\
\pi_{Q, 3} \Delta_{3}=\left(\pi_{Q, 1} \Delta_{1}\right)\left(\frac{L_{1}}{L_{3}}\right)^{\frac{1}{a}}\left(\frac{d_{3}}{d_{1}}\right)^{\frac{b}{a}}\left(\frac{\Delta_{1}}{\Delta_{3}}\right)^{\frac{b-a-3}{a}} \\
\ldots \\
\pi_{Q, n} \Delta_{n}=\left(\pi_{Q, 1} \Delta_{1}\right)\left(\frac{L_{1}}{L_{n}}\right)^{\frac{1}{a}}\left(\frac{d_{n}}{d_{1}}\right)^{\frac{b}{a}}\left(\frac{\Delta_{1}}{\Delta_{n}}\right)^{\frac{b-a-3}{a}} \\
\pi_{Q, 1} \Delta_{1}+\pi_{Q, 2} \Delta_{2}+\ldots+\pi_{Q, n} \Delta_{n}=\pi_{Q} \Delta
\end{gathered} .
$$


By taking into account equation (6), $\pi_{Q, i} \Delta_{i}=Q_{i} \frac{\rho}{\mu}$ and the system of equations (17) may be presented as it follows:

$$
\mid \begin{gathered}
Q_{2}=Q_{1}\left(\frac{L_{1}}{L_{2}}\right)^{\frac{1}{a}}\left(\frac{d_{2}}{d_{1}}\right)^{\frac{b}{a}}\left(\frac{\Delta_{1}}{\Delta_{2}}\right)^{\frac{b-a-3}{a}} \\
Q_{3}=Q_{1}\left(\frac{L_{1}}{L_{3}}\right)^{\frac{1}{a}}\left(\frac{d_{3}}{d_{1}}\right)^{\frac{b}{a}}\left(\frac{\Delta_{1}}{\Delta_{3}}\right)^{\frac{b-a-3}{a}} \\
\ldots \\
Q_{n}=Q_{1}\left(\frac{L_{1}}{L_{n}}\right)^{\frac{1}{a}}\left(\frac{d_{n}}{d_{1}}\right)^{\frac{b}{a}}\left(\frac{\Delta_{1}}{\Delta_{n}}\right)^{\frac{b-a-3}{a}} \\
Q_{1}+Q_{2}+\ldots+Q_{n}=Q
\end{gathered}
$$

For estimating the flow rate $Q_{1}$ in the first pipe the following equation is used:

$$
Q_{1}=\frac{1}{1+\left(\frac{L_{1}}{L_{2}}\right)^{\frac{1}{a}}\left(\frac{d_{2}}{d_{1}}\right)^{\frac{b}{a}}\left(\frac{\Delta_{1}}{\Delta_{2}}\right)^{\frac{b-a-3}{a}}+\ldots+\left(\frac{L_{1}}{L_{n}}\right)^{\frac{1}{a}}\left(\frac{d_{n}}{d_{1}}\right)^{\frac{b}{a}}\left(\frac{\Delta_{1}}{\Delta_{n}}\right)^{\frac{b-a-3}{a}}} Q .
$$

Using the first " $n$ " number of equations in (20) enables the estimation of the other flow rates $Q_{i}$.

For example, the flow rate $Q_{k}$ ensured in the pipe, $\mathrm{k} "(\mathrm{k}=1 \ldots \mathrm{n})$ is estimated by the following way:

$$
Q_{k}=\frac{1}{\sum_{i=1}^{n}\left(\frac{L_{k}}{L_{i}}\right)^{\frac{1}{a}}\left(\frac{d_{i}}{d_{k}}\right)^{\frac{b}{a}}\left(\frac{\Delta_{k}}{\Delta_{i}}\right)^{\frac{b-a-3}{a}}} Q .
$$

The pressure drop between the inlet and outlet (collector points) of the parallel connected pipes is determined by using the system total flow rate $Q$ and the applied method of calculation is described below. First, for analyzing the " $\mathrm{k}$ "th" pipe the relevant criteria equation $\pi_{p k}=A \pi_{Q k}^{a} \pi_{d k}^{b}$ is applied in the following way:

$$
p \frac{\rho \Delta_{k}^{3}}{L_{k} \mu^{2}}=A\left(Q_{k} \frac{\rho}{\mu \Delta_{k}}\right)^{a}\left(\frac{\Delta_{k}}{d_{k}}\right)^{b} .
$$

Then for the pressure drop it may be stated: 


$$
p=\left(A \rho^{a-1} \mu^{2-a}\right) \frac{L_{k}}{d_{k}^{b}} \Delta_{k}^{b-a-3} Q_{k}^{a} .
$$

Taking into account equation (22), concerning the estimation of the flow rate in the " $\mathrm{k}$ "th" pipe, enables the estimation of the pressure drop:

$$
p=\left(A \rho^{a-1} \mu^{2-a}\right) \frac{\frac{L_{k}}{d_{k}^{b}} \Delta_{k}^{b-a-3}}{\left[\sum_{i=1}^{n}\left(\frac{L_{k}}{L_{i}}\right)^{\frac{1}{a}}\left(\frac{d_{i}}{d_{k}}\right)^{\frac{b}{a}}\left(\frac{\Delta_{k}}{\Delta_{i}}\right)^{\frac{b-a-3}{a}}\right]^{a}} Q^{a} .
$$

Transforming mathematically the above equation enables the establishment of the final equation for estimating the pressure drop in case of parallel connected pipes:

$$
p=\left(A \rho^{a-1} \mu^{2-a}\right) \frac{1}{\left[\sum_{i=1}^{n}\left(\frac{d_{i}^{b}}{L_{i}}\right)^{\frac{1}{a}}\left(\frac{1}{\Delta_{i}}\right)^{\frac{b-a-3}{a}}\right]^{a}} Q^{a} .
$$

Finally, taking into account equation (26) enabling the determination of the resistance coefficient of a pipe system, containing " $n$ " number of parallel connected pipes (the exponent is: $\alpha=a$ ):

$$
k=\left(A \rho^{a-1} \mu^{2-a}\right) \frac{1}{\left[\sum_{i=1}^{n}\left(\frac{d_{i}^{b}}{L_{i}}\right)^{\frac{1}{a}}\left(\frac{1}{\Delta_{i}}\right)^{\frac{b-a-3}{a}}\right]^{a}},
$$

\subsubsection{Same type of pipes connected in parallel}

In this case, the absolute equivalent wall roughness will be the same for all the pipes. Therefore:

$$
\Delta_{1}=\Delta_{2}=\ldots=\Delta_{n}=\Delta .
$$

The ratio between the pipes roughness in equation (22) is $\Delta_{k} / \Delta_{i}=1$ and therefore the flow rate in the " $\mathrm{k}$ "th" pipe is given in the following way:

$$
Q_{k}=\frac{1}{\sum_{i=1}^{n}\left(\frac{L_{k}}{L_{i}}\right)^{\frac{1}{a}}\left(\frac{d_{i}}{d_{k}}\right)^{\frac{b}{a}}} Q .
$$

The pressure drop is determined by using the following equation: 


$$
p=\left(A \rho^{a-1} \mu^{2-a}\right) \Delta^{b-a-3} \frac{1}{\left[\sum_{i=1}^{n}\left(\frac{d_{i}^{b}}{L_{i}}\right)^{\frac{1}{a}}\right]^{a}} Q^{a} .
$$

Using equations (26) and (30) enables for the resistance characteristic $p=f(Q)$ of a system, containing " $\mathrm{n}$ " number of parallel connected pipes, to be presented graphically.

\subsection{Systems containing pipes that are connected in series}

In case of a system containing " $m$ " number of serial connected pipes, having different diameters, the following statements are valid $[3,4]$ :

- the total pressure loss is equal to the sum of the pressure losses obtained in each separate pipe:

$$
p=p_{1}+p_{2}+\ldots+p_{m}
$$

- the flow rate in each separate pipe is equal to the system total flow rate:

$$
Q=Q_{1}=Q_{2}=\ldots=Q_{m} \text {. }
$$

Taking into account equation (6) and presenting the flow rate $\mathrm{Q}_{\mathrm{i}}$ by the relevant flow rate criterion $\pi_{Q, i}$ the above equation may be transformed and presented in the following way:

$$
\pi_{Q, 1} \Delta_{1}=\pi_{Q, 2} \Delta_{2}=\ldots=\pi_{Q, m} \Delta_{m}
$$

For determining the pressure loss in each pipe it is necessary to involve equations (7) and (31):

$$
p=\pi_{p 1} L_{1} \frac{\mu^{2}}{\rho \Delta_{1}^{3}}+\pi_{p 2} L_{2} \frac{\mu^{2}}{\rho \Delta_{2}^{3}}+\ldots+\pi_{p m} L_{m} \frac{\mu^{2}}{\rho \Delta_{m}^{3}}=\frac{\mu^{2}}{\rho}\left(\pi_{p 1} \frac{L_{1}}{\Delta_{1}^{3}}+\pi_{p 2} \frac{L_{2}}{\Delta_{2}^{3}}+\ldots+\pi_{p m} \frac{L_{m}}{\Delta_{m}^{3}}\right)
$$

Using the hydraulic criteria equation (1) for presenting the criterions $\pi_{p, i}$ leads to the equation above being presented in the following way:

$$
p=A \frac{\mu^{2}}{\rho}\left(\pi_{Q, 1}^{a} \pi_{d 1}^{b} \frac{L_{1}}{\Delta_{1}^{3}}+\pi_{Q, 2}^{a} \pi_{d 2}^{b} \frac{L_{2}}{\Delta_{2}^{3}}+\ldots+\pi_{Q, m}^{a} \pi_{d m}^{b} \frac{L_{m}}{\Delta_{m}^{3}}\right),
$$

which by replacing the criterions: $\pi_{Q, i}=Q \frac{\rho}{\mu} \frac{1}{\Delta_{i}} ; \pi_{d i}=\frac{\Delta_{i}}{d_{i}}$, and after being mathematically transformed may be presented in the following way:

$$
p=\left(A \rho^{a-1} \mu^{2-a}\right)\left(\Delta_{1}^{b-a-3} \frac{L_{1}}{d_{1}^{b}}+\Delta_{2}^{b-a-3} \frac{L_{2}}{d_{2}^{b}}+\ldots+\Delta_{m}^{b-a-3} \frac{L_{m}}{d_{m}^{b}}\right) Q^{a} .
$$


Equation (36), describing the resistance characteristic of a pipe system containing serial connected pipes, is considered to be more useful after being converted and presented analogically to equation (26):

$$
p=\left(A \rho^{a-1} \mu^{2-a}\right)\left(\sum_{i=1}^{m} \Delta_{i}^{b-a-3} \frac{L_{i}}{d_{i}^{b}}\right) Q^{a} .
$$

Taking into account equation (8) enables for the coefficient of the system resistance characteristic to be estimated by using the following equation:

$$
k=\left(A \rho^{a-1} \mu^{2-a}\right)\left(\sum_{i=1}^{m} \Delta_{i}^{b-a-3} \frac{L_{i}}{d_{i}^{b}}\right) .
$$

The next equation describes the system resistance characteristic in case that it contains of the same type serial connected pipes:

$$
p=\left(A \rho^{a-1} \mu^{2-a} \Delta^{b-a-3}\right)\left(\sum_{i=1}^{m} \frac{L_{i}}{d_{i}^{b}}\right) Q^{a} .
$$

By using equations (37) and (39) the resistance characteristic $p=f(Q)$ of the system, containing " $\mathrm{m}$ " number of serial connected pipes, may be presented graphically.

\subsection{Systems containing combined (parallel and serial) connected pipes}

In case of a system containing " $\mathrm{m}$ " number of serial connected pipes, including a sector of " $n$ " number of parallel connected pipes, the equation describing the system resistance characteristic represents:

$$
p=\left(A \rho^{a-1} \mu^{2-a}\right)\left(\sum_{i=1}^{m} \Delta_{i}^{b-a-3} \frac{L_{i}}{d_{i}^{b}}+\frac{1}{\left[\sum_{i=1}^{n}\left(\frac{d_{i}^{b}}{L_{i}}\right)^{\frac{1}{a}}\left(\frac{1}{\Delta_{i}}\right)^{\frac{b-a-3}{a}}\right]^{a}}\right) Q^{a} .
$$

In case that the analyzed system contains different types of pipes the coefficient of its resistance characteristic may be estimated in the following way: 


$$
k=\left(A \rho^{a-1} \mu^{2-a}\right)\left(\sum_{i=1}^{m} \Delta_{i}^{b-a-3} \frac{L_{i}}{d_{i}^{b}}+\frac{1}{\left[\sum_{i=1}^{n}\left(\frac{d_{i}^{b}}{L_{i}}\right)^{\frac{1}{a}}\left(\frac{1}{\Delta_{i}}\right)^{\frac{b-a-3}{a}}\right]^{a}}\right) .
$$

In case that the analyzed system contains same types of pipes, i.e. $\Delta_{1}=\Delta_{2}=\ldots=\Delta_{n}$, the equation for estimating the system pressure loss, describing its resistance characteristic, represents:

$$
p=\left(A \rho^{a-1} \mu^{2-a}\right) \Delta^{b-a-3}\left(\sum_{i=1}^{m} \frac{L_{i}}{d_{i}^{b}}+\frac{1}{\left[\sum_{i=1}^{n}\left(\frac{d_{i}^{b}}{L_{i}}\right)^{\frac{1}{a}}\right]^{a}}\right) Q^{a} .
$$

Therefore, the coefficient of the system resistance characteristic may be estimated as it follows:

$$
k=\left(A \rho^{a-1} \mu^{2-a}\right) \Delta^{b-a-3}\left(\sum_{i=1}^{m} \frac{L_{i}}{d_{i}^{b}}+\frac{1}{\left[\sum_{i=1}^{n}\left(\frac{d_{i}^{b}}{L_{i}}\right)^{\frac{1}{a}}\right]^{a}}\right) .
$$

\section{Comparative and numerical analysis and results discussion}

\subsection{Output data}

In [2], for determining the exponents by applying equations (1) and (2) the friction factor $\lambda$ is estimated by using the equation of Konakov $-\lambda=\left(1.8 \log \frac{\mathrm{Re}}{6.9}\right)^{-2}$, characterizing hydraulic smooth pipes, while in [3] - for the other regimes of 
motion, the equation of Altschul - $\lambda=0.11\left(\frac{68}{\mathrm{Re}}+\frac{\Delta}{d}\right)^{0,25}$, is used.

A comparative analysis indicates good corresponding between the results found in the previously mentioned works and the results found after applying the proposed methodology, but in case that the friction factor is estimated by using the wellknown and worldwide recognized equation of Colebrook-White [1,4], which requires for the values of the relevant constants and exponents in equations (1) and (2) to be preliminary determined. Table 1 provides information about their values, where the exponents are also presented as simple fractions.

Table 1. Values of the coefficients and exponents belonging to equations (1) and (2)

\begin{tabular}{|c|c|c|c|c|c|c|}
\hline $\mathbf{A}$ & $\mathbf{a}$ & $\mathbf{b}$ & & $\mathbf{B}$ & $\mathbf{e}$ & $\mathbf{f}$ \\
\hline 0.137 & 1.96 & 5.18 & & 2.75 & 0.51 & 2.64 \\
\hline & $45 / 23$ & $119 / 23$ & & & $23 / 45$ & $119 / 45$ \\
\hline
\end{tabular}

Fig.1 illustrates a scheme of a pipe system, containing combined connected pipes, which is analyzed by applying the methodology, newly established by the authors, as for this aim the required information concerning the system parameters is given in Table 2. The flow rate is considered to be: $Q=40 \mathrm{l} / \mathrm{s}$.

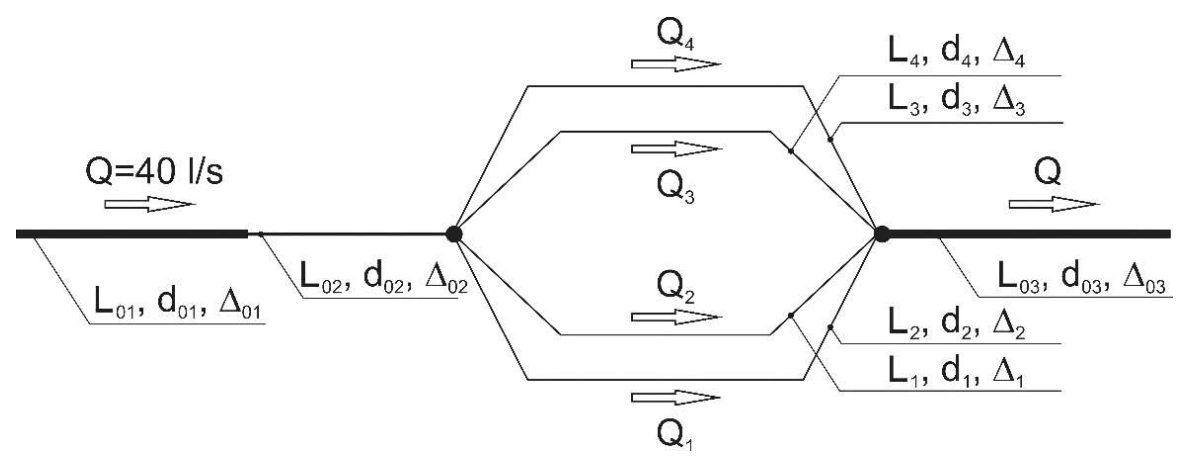

Fig. 1. A scheme of the analysed pipe system.

Table 2. Geometric characteristics of the key elements of the analyzed pipe system

\begin{tabular}{|c|c|c|c|c|c|c|}
\hline \multirow{2}{*}{ Pipe } & \multicolumn{2}{|c|}{ Diameter } & \multicolumn{2}{c|}{$\begin{array}{c}\text { Pipe referred } \\
\text { length }\end{array}$} & \multicolumn{2}{c|}{ Wall roughness } \\
\cline { 2 - 7 } & \multicolumn{2}{|c|}{$[\mathbf{m m}]$} & \multicolumn{2}{|c|}{ [m] } & \multicolumn{2}{c|}{ [mm] } \\
\hline 01 & $\mathrm{~d}_{01}$ & 160 & $\mathrm{~L}_{01}$ & 150 & $\Delta_{01}$ & 0.05 \\
\hline 02 & $\mathrm{~d}_{02}$ & 150 & $\mathrm{~L}_{02}$ & 40 & $\Delta_{02}$ & 0.1 \\
\hline
\end{tabular}




\begin{tabular}{|c|c|c|c|c|c|c|}
\hline 1 & $\mathrm{~d}_{1}$ & 80 & $\mathrm{~L}_{1}$ & 100 & $\Delta_{1}$ & 0.05 \\
\hline 2 & $\mathrm{~d}_{2}$ & 70 & $\mathrm{~L}_{2}$ & 50 & $\Delta_{2}$ & 0.1 \\
\hline 3 & $\mathrm{~d}_{3}$ & 60 & $\mathrm{~L}_{3}$ & 40 & $\Delta_{3}$ & 0.1 \\
\hline 4 & $\mathrm{~d}_{4}$ & 90 & $\mathrm{~L}_{4}$ & 120 & $\Delta_{4}$ & 0.05 \\
\hline 03 & $\mathrm{~d}_{03}$ & 160 & $\mathrm{~L}_{03}$ & 200 & $\Delta_{03}$ & 0.1 \\
\hline
\end{tabular}

The object of this research respectively is to determine: the flow rate ensured in each of the parallel connected pipes - $Q_{1}, Q_{2}, Q_{3}$ and $Q_{4}$; the system pressure drop $p$; the equation of the system resistance characteristic (8) - $p=k Q^{\alpha}$.

\subsection{Results found by using the proposed criteria equations}

Taking into account the values, given in Table 1, concerning the exponents and constants belonging to equations (1) and (2), the required equations for solving the previously considered tasks are being transformed into the following way:

- Parallel connected pipes

Equation (22), applied in estimating the ensured flow rate $Q_{k}$ in the , $\mathrm{k}^{\text {th }}$, pipe ( $k=1 \ldots 4$ ), represents:

$$
Q_{k}=\frac{1}{\sum_{i=1}^{n}\left(\frac{L_{k}}{L_{i}}\right)^{\frac{23}{45}}\left(\frac{d_{i}}{d_{k}}\right)^{\frac{119}{45}}\left(\frac{\Delta_{k}}{\Delta_{i}}\right)^{\frac{1}{9}}} Q .
$$

By applying equation (26) it may be determined the pressure drop $p_{p}$ in case of parallel connected pipes:

- Serial connected pipes:

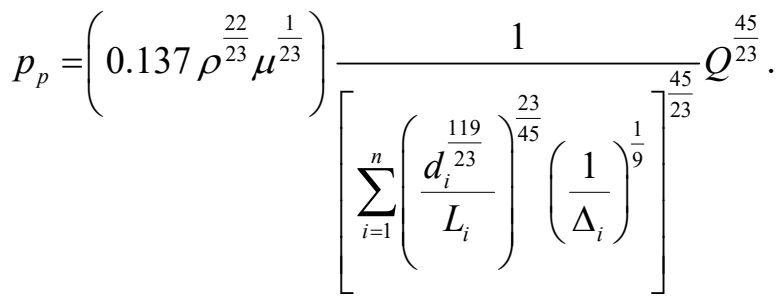

The pressure drop $p_{s}$ in case of serial connected pipes is estimated in accordance with equation (37):

$$
p_{S}=\left(0.137 \rho^{\frac{22}{23}} \mu^{\frac{1}{23}}\right)\left(\sum_{i=1}^{3} \Delta_{i}^{\frac{5}{23}} \frac{L_{i}}{d_{i}^{\frac{119}{23}}}\right) Q^{\frac{45}{23}} .
$$

Equation (40), applied for a system containing combined connected pipes, is 
used to determine its resistance characteristic (8):

$$
p=\left(0.137 \rho^{\frac{22}{23}} \mu^{\frac{1}{23}}\right)\left(\sum_{i=1}^{3} \Delta_{i}^{\frac{5}{23}} \frac{L_{i}}{d_{i}^{\frac{119}{23}}}+\frac{1}{\left[\sum_{i=1}^{4}\left(\frac{d_{i}^{\frac{119}{23}}}{L_{i}}\right)^{\frac{23}{45}}\left(\frac{1}{\Delta_{i}}\right)^{\frac{1}{9}}\right]^{\frac{45}{23}}}\right) Q^{\frac{45}{23}} .
$$

The results found are given in Table 3 .

The final equation of the system resistance characteristic represents:

$$
p=81134454.5 Q^{\frac{45}{23}} \text {. }
$$

\subsection{Results found after the specialized software "Pipe Flow Expert" is used}

The specialized software product "PipeFlow Expert" [5] has a powerful and reliable computing "engine" containing preliminary added algorithms, based on well-known physical relations that are published in many research works concerning problems in industry, as well as particular algorithms and methodologies established and developed (in addition) by the British company "Daxesoft" Ltd.

For the aims of this current work it is used a licensed version of the above mentioned software product, which is donated by the company "Daxesoft" Ltd. to the University "Angel Kanchev" of Ruse and more specifically to the dept. of "Heat, Hydraulics and Environmental Engineering".

By involving accurately the output data concerning the analyzed pipe system key elements: lengths, diameters, wall roughness and the ensured flow rate $Q$, it is possible to design its computational scheme, given in Fig.2.

The numerical computation is accomplished under the following conditions:

- Calculation Engine:

- Method of Solution: Non-Compressible Flow [Accurate];

- Method of Calculation: Darcy-Weisbach Equation;

- Friction Model: Colebrook-White Friction Factors;

- Results found:

- Found linear solution flow values. Refining...

- Final solution iterations: 2.

- Verifying pipe losses against node pressures at start and end of pipe. 


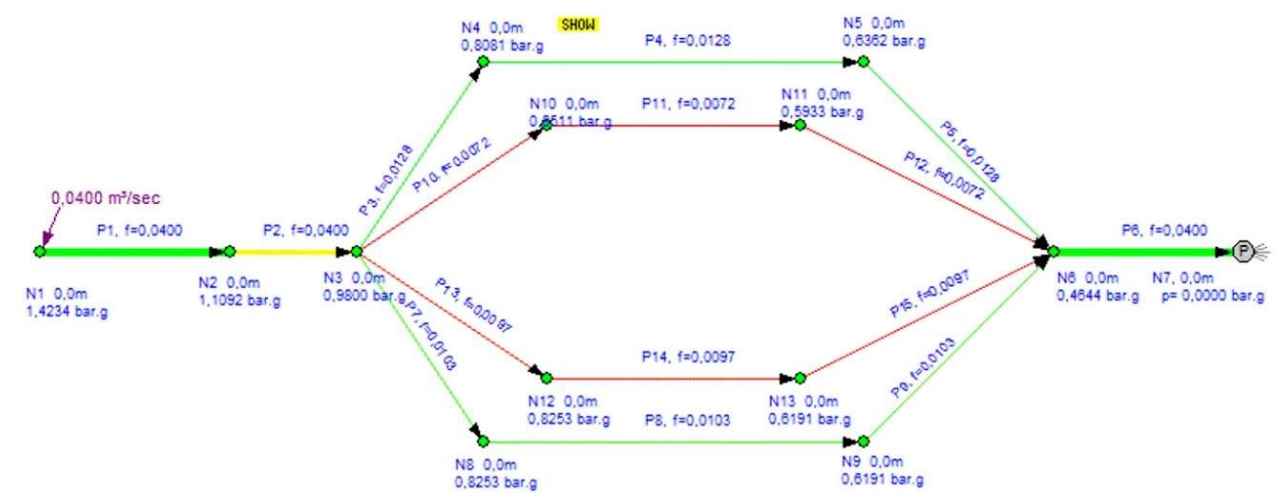

Fig. 2. Computational scheme of the analyzed pipe system designed and solved by using "Pipe Flow Expert".

The final results are given in Table 3.

Table 3. Comparative analysis results

\begin{tabular}{|c|c|c|c|c|c|c|c|c|}
\hline \multirow[t]{3}{*}{ Pipe } & \multicolumn{4}{|c|}{ By using criteria equations } & \multicolumn{4}{|c|}{ By using "Pipe Flow Expert" } \\
\hline & \multicolumn{2}{|c|}{ Flow rate } & \multicolumn{2}{|c|}{ Pressure drop } & \multicolumn{2}{|c|}{ Flow rate } & \multicolumn{2}{|c|}{ Pressure drop } \\
\hline & \multicolumn{2}{|c|}{$\left[\mathbf{m}^{3} / \mathbf{s}\right]$} & \multicolumn{2}{|c|}{$[\mathrm{Pa}]$} & \multicolumn{2}{|c|}{$\left[\mathrm{m}^{3} / \mathbf{s}\right]$} & \multicolumn{2}{|c|}{$[\mathrm{Pa}]$} \\
\hline 01 & $Q_{01}$ & 0,040 & $p_{01}$ & 31807,8 & $Q_{01}$ & 0,040 & $p_{01}$ & 31420 \\
\hline 02 & $Q_{02}$ & 0,040 & $p_{02}$ & 13770,9 & $Q_{02}$ & 0,040 & $p_{02}$ & 12920 \\
\hline 1 & $Q_{1}$ & 0,01036 & $p_{1}$ & 54429,4 & $Q_{1}$ & 0,0103 & $p_{1}$ & 51560 \\
\hline 2 & $Q_{2}$ & 0,00960 & $p_{2}$ & 54429,4 & $Q_{2}$ & 0,0097 & $p_{2}$ & 51560 \\
\hline 3 & $Q_{3}$ & 0,00716 & $p_{3}$ & 54429,4 & $Q_{3}$ & 0,0072 & $p_{3}$ & 51560 \\
\hline 4 & $Q_{4}$ & 0,01288 & $p_{4}$ & 54429,4 & $Q_{4}$ & 0,0128 & $p_{4}$ & 51560 \\
\hline 03 & $Q_{04}$ & 0,040 & $p_{03}$ & 49307,6 & $Q_{04}$ & 0,040 & $p_{03}$ & 46440 \\
\hline \multicolumn{4}{|c|}{ Total system pressure drop } & 149316 & \multicolumn{3}{|c|}{ Total system pressure drop } & 142340 \\
\hline
\end{tabular}

The analysis of the results found clearly indicates sufficient overlapping in using both the selected methods of calculation, since the values concerning the analyzed parameters are practically equal. This is an indirect indicator confirming the accurate using of these newly established equations in engineering calculations concerning pipe systems.

It may be stated that, the accomplishing of a hydraulic analysis of pipe systems by applying the classical methods of calculation leads to the obtaining of inaccuracies that in some cases exceed $5 . .6 \%$. The inaccurate determining of the 
pipe wall roughness has most significant impact on this process, while it is less influenced by the equations used for estimating the friction factor and local resistance coefficients. In this regard, the discrepancy between the results found by using the newly established criteria equations and "Pipe Flow Expert" is considered to be insignificant (negligible) which confirms the great potential in applying this methodology in accomplishing engineering calculations concerning fluid transporting systems.

\section{Conclusion}

The proposed in this work original methodology, newly established by the authors, to accomplish hydraulic analysis of typical pipe systems contrasts with the classical methods of calculations used as a basis in developing the modern software products. The established equations are characterized by simple mathematical processing enabling them to be easily applicable in finding a solution of many engineering problems. As an important particularity in using the criteria equations (4) and (5) it is considered to be the fact that there is no need to perform all the required steps concerning the accomplishment of such hydraulic estimations, including the determining of the regime of motion (Reynolds number), selecting an appropriate equation for estimating the friction factor, etc. In addition to that, applying the proposed methodology excludes the necessity of searching for an iterative determination of the ensured flow rate, which in case of a more complex pipe system is one of the most laborious tasks, because most often the final solution is found after assuming that the regimes of motion are fully turbulent. Taking into account the above statements, the methodology, proposed in this work, may be considered as a new approach at accomplishing the hydraulic analysis of pipe systems, used to transport fluids.

The equations, newly established by the authors, concerning the determination of the pipe system resistance characteristics are considered to be especially useful in engineering practice and more specifically: in determining a hydraulic machine work regime, used in a system transporting fluids, as well as in the synthesis and analyzing of such systems or in accomplishing an energy analysis of pipe systems. However, the method derived in this work has the following limitations: cannot be applied for looped pipe networks, neither for branched pipe networks; cannot be applied in case of involving one or several inflows and many outflows (end-users).

Acknowledgements: This paper contains results found after working on a project 2020FAI-02, funded by the Fund "Scientific research" of the University of "Angel Kanchev" of Ruse, Bulgaria. 


\section{References}

1. Eugene Adiutori. Why the Fluid Friction Factor should be Abandoned, and the Moody Chart Transformed. The Open Mechanical Engineering Journal, 2009, pp 43-48

2. Popov, Gencho. Hydraulic Criteria Equations for Pipelines Design //PROCEEDINGS of the University "A. Kanchev" of Ruse, volume 50, book 1.2, Ruse, 2011, pp. 54-59

https://www.researchgate.net/publication/312531977_Kriterialni uravnenia za hidravlicno presmatane na trboprovodi_Hydraulic_Criteria_Equations for Pipelines_Design

3. Altshul, A., L. Zhivotobski, L. Ivanov, Hydravlika i aerodinamika. Moskva, 1987

4. White, F. M. Fluid Mechanics. Fourth Edition. McGraw-Hill, 1998.

5. https://www.pipeflow.com/ 